\title{
Aproximación a una metodología de evaluación de la calidad de geoportales IDE
}

\author{
Antonio F. Rodríguez \\ Paloma Abad* \\ Alejandra Sánchez ${ }^{*}$ \\ Marta Juanatey* \\ Ana Cevidanes**
}

Recibido el 15 de marzo de 2017; aceptado el 29 de junio de 2017

\begin{abstract}
In this article, we create a methodology for the verification of the quality of SDI geoportals, paying special attention to usability and interoperability in a broad sense, and we apply it of the SDI geoportals of America. This methodology is based on previous studies and the experience gained in the SDI team of CNIG (National Centre for Geographic Information) Spain.

Until now a big attention and considerable efforts have been dedicated to standardize the web services of a SDI, but we think that little attention has been paid to the geoportals and client applications exploiting those services. Therefore, it would be wise now to try to define objective criteria for the evaluation of the usability and interoperability of geoportals, in order to contribute to its optimization with technical criteria oriented to improve its general quality.

We will try to define a general indicator of SDI geoportal quality defining a set of convenient individual indicators stablished as a result of the experience and knowledge of a group of experts in the field of SDIs.

In this article, we inform about the results obtained applying this methodology to the main national and supranational SDI geoportals of the Americas in order to evaluate its usefulness, to identify improvement opportunities and to contribute to

* Centro Nacional de Información Geográfica (CNIG), Instituto Geográfico Nacional (IGN) de España, Calle General Ibáñez de Ibero, 328003 Madrid, España, correos electrónicos: afrodriguez@fomento.es, pabad@fomento.es, asmagan-to@fomento.es

** Departamento de Ingeniería Topográfica y Cartografía, Universidad Politécnica de Madrid, Calle Mercator 1, Madrid, España, correo electrónico: cevi.topo@gmail.com
\end{abstract}


its optimization. Finally, we outline some conclusions trying to balance the effort and resources needed and the benefits of the possible corrective actions derived of the performed analysis. As a consequence, it would be possible to design an Action Plan prioritizing the activities with a bigger impact in the general quality of geoportals and a lower cost.

Key words: geoportal, usability, interoperability, Spatial Data Infrastructure, web services.

\section{Resumo}

Este artigo apresenta uma metodologia para avaliar a qualidade de um geoportal IDE, com particular atenção à sua usabilidade e interoperabilidade, em sentido amplo e aplicado aos geoportais nacionais das IDE das Américas. Esta metodologia baseia-se em documentos de trabalho anteriores e na experiência acumulada pela equipe IDE do CNIG (Centro Nacional de Informação Geográfica) da Espanha.

Até o momento, muita atenção foi dada e um esforço considerável foi feito para padronizar os serviços no contexto das IDE mas, acredita-se que tenha sido dada pouca atenção aos geoportais e clientes que exploram esses serviços. Portanto, parece oportuno abordar critérios objetivos de usabilidade e interoperabilidade de geoportais que contribuam para otimizá-los a partir de critérios técnicos e objetivos que tendem a melhorar sua qualidade geral.

Se tentará definir um indicador de qualidade geral de geoportal IDE que defina um conjunto de indicadores individuais, cuja conveniência e oportunidade derivam da experiência e do conhecimento de um grupo de especialistas no campo das IDE.

Este artigo relata os resultados obtidos a partir da aplicação da metodologia definida aos geoportais de referência nacionais das Infraestruturas de Dados Espaciais do continente americano para avaliar sua utilidade prática, identificar áreas para melhoria e contribuir para sua otimização. Finalmente, são feitas uma série de considerações que tentam equilibrar o custo e o resultado das possíveis medidas corretivas derivadas da análise realizada. Esta análise permite sugerir amplamente um plano de implementação que aborda as medidas de menor custo e maior impacto na qualidade geral dos recursos gerenciados.

Palavras-chave: geoportal, usabilidade, interoperabilidade, infraestrutura de dados espaciais, serviços web.

\section{Resumen}

En este artículo se plantea una metodología de evaluación de la calidad de un geoportal de una IDE, dedicando especial atención a su usabilidad e interoperabilidad, entendidas en sentido amplio, y se aplica a los geoportales nacionales de las IDE de las Américas. Esta metodología está basada en documentos anteriores de trabajo y 
la experiencia acumulada en el equipo IDE del CNIG (Centro Nacional de Información Geográfica) de España.

Hasta ahora se ha dedicado mucha atención y se ha invertido un notable esfuerzo de estandarización de servicios en el contexto de las IDE, pero creemos que se ha prestado poca atención a los geoportales y clientes que explotan dichos servicios. Por eso, nos parece oportuno abordar criterios objetivos de usabilidad e interoperabilidad de geoportales que contribuyan a optimizarlos desde criterios técnicos y objetivos que atiendan a mejorar su calidad general.

Se tratará de definir un indicador de calidad general de un geoportal IDE definiendo un conjunto de indicadores individuales cuya conveniencia y oportunidad se deriva de la experiencia y conocimiento de un grupo de expertos en el campo de las IDE.

En este artículo se informa de los resultados obtenidos de la aplicación de la metodología definida a los geoportales de referencia nacionales de las Infraestructuras de Datos Espaciales del continente americano para evaluar su utilidad práctica, identificar áreas de mejora y contribuir a su optimización. Finalmente se realiza una serie de consideraciones que tratan de equilibrar el coste y el resultado de las posibles medidas correctivas derivadas de este análisis. El estudio permite sugerir a grandes rasgos un proyecto de implantación que aborda primero las medidas de menor coste y mayor impacto en la calidad general de los recursos gestionados.

Palabras clave: geoportal, usabilidad, interoperabilidad, Infraestructura de Datos Espaciales, servicios web.

\section{Introducción}

Tal y como se puede comprobar en la literatura existente (Manrique y Manso, 2012) hasta ahora, el énfasis en la estandarización y verificación de conformidad necesarias para lograr que las Infraestructuras de Datos Espaciales (IDE) alcancen sus objetivos, se ha realizado en la parte servidora. Efectivamente se ha invertido un notable esfuerzo en la normalización y estandarización de los servicios web de datos geográficos, datos y metadatos, sobre los que recae la mayor presión normalizadora de las normas ISO 19100 sobre servicios, los estándares OGC y, en Europa, la Directiva INSPIRE (Comisión Europea, 2007) y los Reglamentos asociados:

- En las normas ISO 19100 de información geográfica dedicadas a servicios, tan solo la norma ISO 19128 (2005) sobre WMS contempla un Abstract Test Suite (ATS) para servicios y visualizadores, es decir un procedimiento para verificar objetivamente el cumplimiento de la norma y poder certificarla conformidad, tanto para la parte cliente como para la servidora. El resto de normas, ISO 19133 (2005) e ISO 19134 (2007) sobre Location Based Services, y la ISO 
19142 (2010) sobre WFS incluyen un ATS que considera exclusivamente a la parte servidora.

- En cuanto a las especificaciones OGC que definen interfaces de servicio, los únicos estándares que consideran en su ATS a la parte cliente son OGC WMS (2006), OGC WMTS (2012), OGC WSC (2010) y OGC SoS (2012). El resto de especificaciones OGC tienen como objetivo estandarizar el servicio y no la interfaz, lo que incluiría también a la parte cliente.

- También resulta significativo que el OGC puso a disposición de los desarrolladores el primer test de conformidad para software cliente en junio del 2015, ${ }^{1}$ ciertamente una fecha relativamente reciente.

- En cuanto a las Normas de Ejecución ${ }^{2}$ de la Directiva Europea INSPIRE (Comisión Europea, 2007), sólo contemplan la estandarización de servicios, toda vez que la Directiva, los reglamentos que la implementan y todo el cuerpo normativo se aplican tan solo a la parte servidora.

En el mismo sentido, las principales aplicaciones disponibles de verificación de estándares de interoperabilidad en el campo de las IDE se centran en la parte servidora.

Sin embargo, en nuestra opinión, se ha prestado apenas atención a la parte cliente, si exceptuamos los estudios clásicos de usabilidad (Manrique y Manso, 2012), quizás debido en cierta medida al hecho de que la mayoría de los estándares de servicio son aplicables a servidores y sólo cuando se adoptan como normas ISO van acompañados de un Conjunto de Pruebas Genéricas (Abstract Test Suite) aplicables a la parte cliente.

En realidad, nada nuevo se está exigiendo a los geoportales, salvo un cierto nivel de utilidad, usabilidad y accesibilidad, por ser el entorno en el que tienen lugar las actividades de búsqueda interactiva de datos y servicios, consulta, análisis y, cuando es posible, descarga de datos. Creemos que estos aspectos de los geoportales son clave porque a través de ellos los usuarios desarrollan las actividades de consulta y explotación interactivas, y los desarrolladores buscan los recursos que necesitan como paso previo a la programación de la aplicación que explota esos recursos de manera automática o semiautomática.

Por todo ello, nos parece oportuno en el momento actual, en el que las IDE de las Américas florecen y ofrecen un buen número de servicios web de información geográfica con un grado de madurez razonable y creciente, abordar en este número especial dedicado a los geoportales, criterios objetivos de usabilidad e interoperabi-

$<$ http://www.opengeospatial.org/blog/2244>.

$<$ http://inspire.ec.europa.eu/network-services/41>. 
lidad de geoportales que contribuyan a optimizarlos desde criterios técnicos y objetivos que atiendan a su eficacia y facilidad de uso.

\section{Objetivos}

Los objetivos que atiende este artículo son los siguientes:

- Definir un conjunto de criterios, con sus respectivos indicadores para caracterizar la calidad general, en cuanto a usabilidad e interoperabilidad, entendidas en sentido amplio, de los geoportales IDE desde un punto de vista práctico, según la experiencia del equipo IDE del CNIG en este campo.

- Aplicarlos al caso de los geoportales nacionales y supranacionales del continente americano, para obtener una visión global del estado de la cuestión y poder extraer así criterios y pautas generales sobre su mejora.

- Extraer conclusiones generales de los resultados y resaltar los patrones o tendencias que se consideren relevantes y significativas.

Lamentablemente, apenas si se han encontrado antecedentes o referencias significativas en literatura sobre calidad de geoportales, más allá de los estudios de usabilidad realizados. Por ese motivo, se ha optado por basar esta metodología en la experiencia del equipo IDE del IGN, contrastada mediante discusiones y debates en el seno del Grupo Técnico de la IDEE y en el desarrollo de cursos y seminarios (Rodríguez et al., 2008).

\section{Definiciones}

Para el desarrollo de este trabajo se aplican las siguientes definiciones:

- Geoportal: sitio Internet o equivalente que proporciona acceso a servicios interoperables de información geográfica. Constituye por lo tanto la cara visible y el punto de entrada a un nodo IDE y por extensión, a la IDE de un cierto ámbito de actuación (LISIGE, 2010).

- Nodo IDE: dominio web en el que una organización publica un conjunto de servicios, clientes y, en general, recursos IDE. No hay un número mínimo de recursos a publicar.

- Infraestructura de Datos Espaciales: sistema de sistemas, que incluye más de un nodo IDE, formado por tecnología, políticas y disposiciones administrativas, cuya finalidad es facilitar el acceso a datos geoespaciales y compartirlos. Esta definición es nuestra y está elaborada a partir del GSDI Cookbook (GSDI, 2012; Béjar et al., 2009). 
- Interoperabilidad: capacidad de comunicar, ejecutar programas o transferir datos entre varias unidades funcionales de manera que requiera del usuario tener un conocimiento pequeño o nulo de las características específicas de esas unidades (ISO/IEC 2382-1:1993).

- Usabilidad: eficacia, eficiencia y satisfacción con la que un producto permite alcanzar objetivos específicos a usuarios específicos en un contexto de uso específico (ISO/IEC 9241-11:1998).

\section{Método de trabajo}

Se han seleccionado un total de 19 criterios que atienden a otros tantos aspectos de la usabilidad e interoperabilidad de geoportales, como resultado de más de nueve años de experiencia (Rodríguez, 2012) y análisis de:

- Los geoportales de la IDE de España para avanzar en su interoperabilidad (Rodríguez et al., 2015).

- Los geoportales de Europa para estudiar el estado de desarrollo de la implementación de la Directiva INSPIRE (Comisión Europea, 2007).

- Los geoportales de América como parte de nuestras actividades de formación en diversos cursos y seminarios (véanse Rodríguez et al., 2008) y para conocer la actividad relacionada con las IDE en el continente (blog-IDEE 2008, 2012 y 2016).

Una vez definidos esos criterios y para obtener una serie de estadísticas y valores globales descriptivos del estado de la cuestión, se han aplicado a un conjunto de 15 geoportales IDE nacionales y un geoportal supranacional del continente americano (Tabla 1). Finalmente, se han formulado algunas conclusiones generales.

Hay que hacer notar que se han considerado tan solo los geoportales nacionales y supranacionales del continente en el sentido antes definido, es decir, sitios web que:

- Albergan al menos un cliente de servicios web de información geográfica, por ejemplo un visualizador.

- Cumplen además la condición de estar operativos en la fecha de referencia que figura en la Tabla 1.

Aunque se han elegido los geoportales de IDE como línea de trabajo más estándar e interoperable en el campo de la información geográfica, este trabajo puede extenderse fácilmente a otro tipo de geoportales no estándar. 
Tabla 1

Geoportales considerados

\begin{tabular}{ll}
\hline \multicolumn{1}{c}{ Proyecto } & \multicolumn{1}{c}{ Dirección URL } \\
\hline Proyecto GEOSUR & $<$ https://www.geosur.info/geosur/index.php/es/> \\
IDE de Argentina & $<$ http://www.idera.gob.ar/> \\
IDE de Bolivia & $<$ http://ideepb.geo.gob.bo/> \\
IDE de Brasil & $<$ http://www.inde.gov.br/> \\
IDE de Canadá & $<$ chttp://www.nrcan.gc.ca/earth-sciences/geomatics/ \\
& $<$ http://www.ide.cl/> \\
IDE de Chile & $<$ http://www.icde.org.co/> \\
IDE de Colombia & $<$ http://www.snitcr.go.cr/> \\
IDE de Costa Rica & $<$ http://sni.gob.ec/> \\
IDE de Ecuador & $<$ http://www.cnr.gob.sv/geoportal-cnr/> \\
IDE de El Salvador & $<$ http://www.segeplan.gob.gt/nportal/index.php/servicios/ \\
IDE de Guatemala & sistemas-en-linea/sinit> \\
IDE de Estados Unidos & $<$ https://www.geolatform.gov/> \\
IDE de Panamá & $<$ http://www.ipde.gob.pa/> \\
IDE de Perú & $<$ http://www.geoidep.gob.pe/> \\
IDE de Uruguay & $<$ http://ide.uy/> \\
IDE de Venezuela & $<$ http://www.geoportalsb.gob.ve/> \\
\hline
\end{tabular}

Fuente: Elaboración propia sobre consultas en la web verificadas el 13 de marzo de 2017.

La mayoría de los criterios considerados, se han verificado simplemente visitando los geoportales en estudio. Se ha realizado una evaluación doble, siempre ejecutada por dos personas, que han tratado de reducir al mínimo el impacto de la subjetividad, aunque hay que reconocer que en un buen número de ocasiones resulta muy difícil por la variedad de criterios que se pueden aplicar a la hora de implementar un requisito determinado.

En cualquier caso, en este trabajo se recopilan los datos estadísticos y se muestran los resultados agregados, sin mencionar en ningún caso algún geoportal en particular. Se ha decidido proceder así por varias razones: lo que se considera interesante es el estado global de la cuestión, no la anécdota de un geoportal en particular; según nuestra experiencia, los estímulos negativos no resultan muy constructivos porque minan el optimismo y fomentan la desconfianza, dos tendencias que hay que evitar en una comunidad como la de los actores que colaboran en una IDE, que debe estar basada en la confianza y el compartir, y hacerlo establece- 
ría comparaciones odiosas, ya que no en todos los casos se ha dispuesto de los mismos recursos, ni las circunstancias son siempre comparables.

En cualquier caso, el presente trabajo se realiza con ánimo de ejercer la crítica constructiva y con la intención de ayudar a mejorar, en la línea de colaboración con los geoinstitutos e instituciones supranacionales del sector en el continente americano que el Centro Nacional de Información Geográfica y el Instituto Geográfico Nacional de España vienen desarrollando desde hace ya un buen número de años.

\section{Criterios considerados}

\section{Apertura}

El acceso al geoportal principal de una IDE debe ser libre, abierto, sin necesidad de que el usuario se registre, se identifique, descargue o instale ningún plug-in (instalable) más allá de los habituales que prácticamente forman parte en cada momento de la configuración estándar del navegador, ya que el objetivo de un geoportal de este tipo es dar la máxima difusión posible a los recursos IDE de su ámbito y cualquiera de las circunstancias mencionadas constituye una barrera ante la que un cierto tanto por ciento de los usuarios desiste.

Este criterio se comprueba simplemente consultando los geoportales de estudio y se ha verificado que se cumple a plena satisfacción en el caso de los 16 geoportales analizados. Tan sólo en algún caso es necesario disponer de algún plug-in concreto, pero como decíamos se trata de un instalable de uso tan extendido que la mayoría de los usuarios ya lo tienen instalado.

\section{Multilingüismo}

Teniendo en cuenta que al publicar un recurso en internet se está actuando en un entorno global e internacionalizado, es requisito de un geoportal IDE que muestre una interfaz multilingüe al menos en el idioma local y en inglés. Incluso se podría esperar una interfaz para cada uno de los idiomas oficiales en cada país y para los idiomas de los países limítrofes, si existen y son diferentes.

Hay que pensar además que la situación más habitual es el multilingüismo, los países que tienen un único idioma oficial en todo su territorio son una excepción. Una política aconsejable puede ser diseñar una interfaz visualmente limpia, con poco texto, fácil de traducir y de mantener traducida, de manera automática o semiautomática, y publicar los textos largos que se desee en forma de documentos que no entorpezcan este aspecto.

En este punto la situación es que de los 16 geoportales considerados, tan solo tres presentan una interfaz multilingüe, es decir en más del $81 \%$ no tiene en cuenta el multilingüismo (véase la Figura 1). 


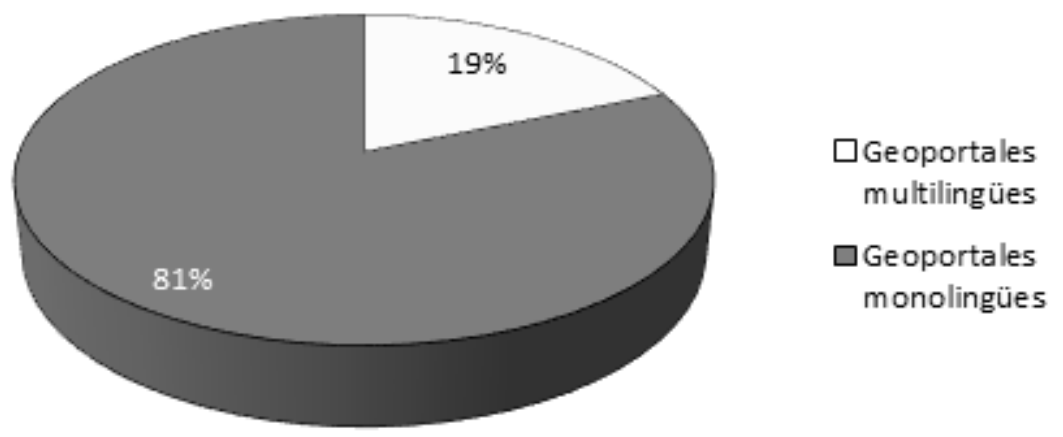

Figura 1. Geoportales IDE multilingües y monolingües en el continente americano.

\section{Responsividad}

Se entiende por responsividad o adaptabilidad de diseño, a la cualidad que tienen los sitios web de adaptarse bien al dispositivo desde el que se les consulta, ya sea un móvil inteligente, una tableta, una televisión digital o cualquier otro. Este criterio cada vez más importante desde que a finales del 2015, el número de móviles en nuestro planeta superó al de habitantes humanos.

Para verificar este aspecto, se han intentado abrir desde un teléfono Android la página web y el visualizador principal de cada nodo IDE. El resultado ha sido que en 12 de los 16 casos, ambas cosas funcionaban correctamente, es decir que el 75\% de los nodos son responsivos (véase la Figura 2).

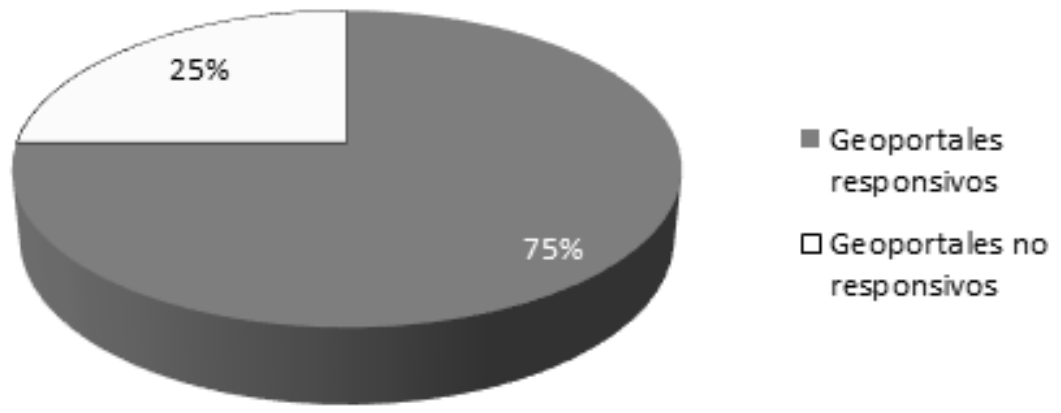

Figura 2. Geoportales IDE responsivos y no responsivos en el continente americano. 


\section{Navegadores}

Otro criterio lógico, que define el Esquema Nacional de Interoperabilidad Español (BOE, 2010) en forma de Principio de Neutralidad Tecnológica, es el que establece que no se debe discriminar al usuario en función de la solución tecnológica por él elegida. Por lo tanto, consiste en esperar que un geoportal IDE exhiba toda su funcionalidad sin limitaciones en los navegadores más extendidos, al menos en las últimas versiones estables de Internet Explorer, Mozilla Firefox, Google Chrome y Microsoft Edge.

La verificación ha consistido en abrir tanto la página principal de cada geoportal como su visualizador en los cuatro navegadores más extendidos y estables, con el resultado de que el $100 \%$ de los geoportales examinados satisface completamente este criterio.

\section{Autodefinición}

Un geoportal debe autodefinirse como un recurso IDE, autodeclararse claramente y sin ambigüedades que se basa en servicios estándar e interoperables, utilizando en un lugar preferente y muy visible alguna de las palabras clave adecuadas cuando sean aplicables (IDE, geoportal, servicios web, estándar, OGC...). Este punto es especialmente relevante para geoportales de IDE nacionales y supranacionales, una de cuyas principales funciones es erigirse como entrada principal y portal de referencia para encontrar los recursos interoperables de su ámbito.

Este criterio se ha verificado consultando los geoportales de estudio, se han encontrado geoportales IDE en cuyo título se emplea el acrónimo SIG o SIT y en los que no hay ninguna referencia a las palabras clave que lo identifican como parte constituyente de una IDE; al contrario, también hay geoportales que se autoproclaman como nodos IDE sin serlo realidad.

De los 16 geoportales IDE analizados, en 13 de ellos, es decir el 81\%, se satisface este requisito de manera clara y evidente, mientras que en los otros tres, creemos que un usuario ingenuo o poco experto puede tener dudas razonables de si se trata de un nodo IDE o no (véase la Figura 3), ya que no se declara así explícitamente y es necesario deducirlo del tipo de servicios que se ofrece en ellos. 


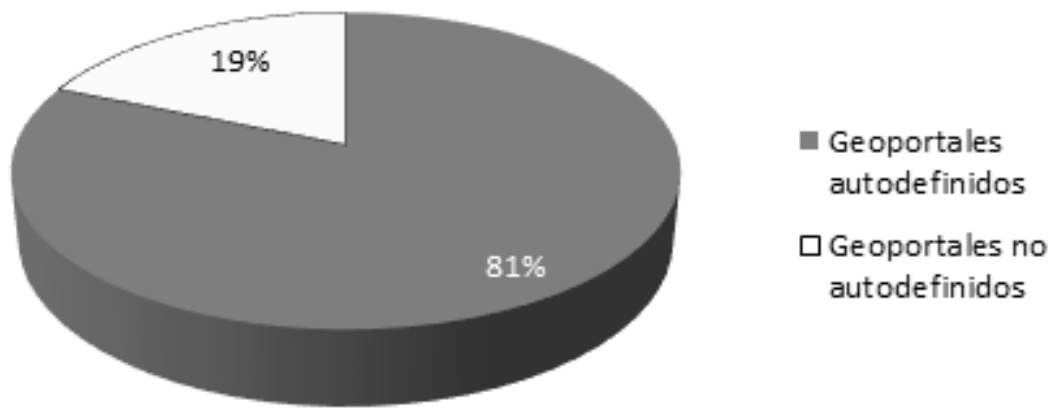

Figura 3. Geoportales que se autodefinen como recursos IDE y los que no lo hacen, en el continente americano.

\section{Identidad}

Una buena parte de los usuarios de Internet otorgan credibilidad a los datos que encuentra en función de la reputación de la persona u organismo responsable de su publicación (Freire, 2010). En ese sentido, pensamos que resulta de la máxima importancia que la organización responsable de la implementación y mantenimiento de un geoportal de referencia se identifique claramente en sus páginas.

De 16 geoportales analizados, en el $93 \%$ de los casos se cumple este requisito.

\section{Dominio}

Un criterio que aplica la aplicación Service Status Checker ${ }^{3}$ del Federal Geographic Data Committee (FGDC) de Estados Unidos para evaluar servicios web es que el nombre del dominio, o primer segmento de la dirección URL, del servicio debe identificar o bien al organismo responsable de su mantenimiento o bien al proyecto en el que se enmarca y en cualquier caso debe servir para encontrar una página en la que se documente una de las dos cosas.

Extrapolando ese criterio a la dirección URL del geoportal, se establece una variante del criterio anterior. Si tomamos el primer segmento de las 16 direcciones URL de los geoportales considerados, en todos los casos se accede a una página que se identifica claramente con el proyecto. 


\section{Accesibilidad}

La accesibilidad o facilidad de acceso de todo tipo de usuarios a un recurso, se está convirtiendo cada vez más en un requisito de obligada consideración en todas las aplicaciones y entornos. Esto es aplicable al caso de recursos web, como los geoportales, independientemente de las limitaciones visuales y sensoriales del usuario.

En ese sentido y dadas las dificultades intrínsecas de accesibilidad que tienen los visualizadores de información geográfica debido al tipo de datos que muestran, nos parece que un mínimo exigible para un geoportal IDE sería que, al menos, las páginas estáticas del geoportal alcanzaren el nivel AA (accesibilidad normal o media). Este nivel está definido por la Iniciativa de Accesibilidad en la Web (WAI) ${ }^{4}$ del consorcio W3C. Y en ese caso, sería esperable que se diera publicidad al cumplimiento del test AA, por lo que para evaluar este aspecto bastaría comprobar qué geoportales muestran el logo de haber superado el nivel AA de la WAI.

De los 16 geoportales analizados, sólo uno tiene un certificado de accesibilidad y los 15 restantes no alcanzan, en nuestra opinión, el nivel mínimo que sería exigible en este aspecto (véase la Figura 4).

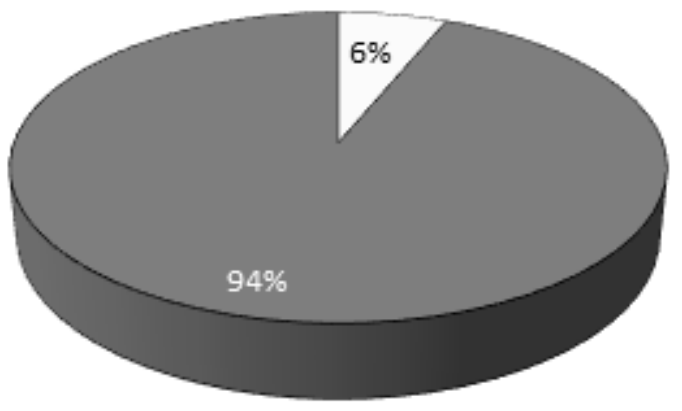

Geoportales con certificado

$\square$ Geoportales no certificados

Figura 4. Geoportales con certificado de accesibilidad en el continente americano.

\section{Usabilidad}

La usabilidad, o facilidad de uso, entendida tal y como se define en la Norma ISO/IEC 9126-1 (ISO/IEC, 2001), es uno de los requisitos que con mayor exigencia se impone a cualquier interfaz que deba ser utilizada por los usuarios (Bernabé y González, 2014; Calderón et al., 2014). En ese sentido, y aunque habría que llevar a cabo un análisis completo y riguroso de usabilidad de los geoportales considerados, lo que rebasa el ámbito de este artículo, se ha hecho una primera evaluación provi- 
sional en la que se ha valorado si el geoportal disponía de un mapa del sitio web o no, el número de clics necesario para llegar al visualizador y si la página tenía o no algún certificado de usabilidad.

Los resultados pueden verse en la Figura 4, que muestra que de 16 geoportales analizados, ninguno exhibe o referencia un estudio o certificado de usabilidad, uno presenta una página especialmente confusa, en tres de ellas (incluyendo a la anterior) es necesario hacer click tres veces para llegar al visualizador, lo que puede ser una barrera real en un teléfono móvil con baja velocidad o en situaciones de mala conexión, y sólo 6 ofrecen al usuario un mapa de la web, por lo que se puede decir que sólo un 37.5 \% (es decir, seis) de los geoportales considerados, satisfacen mínimamente este requisito (véase la Figura 5).

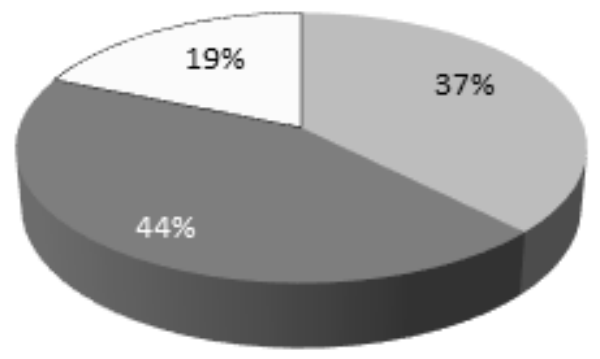

Geoportales con mapa de la web

Geoportales sin mapa de la web y visualizador a menos de 3 clics

$\square$ Geoportales sin mapa web y visualizador a 3 o más clics

Figura 5. Geoportales más y menos usables en el continente americano.

\section{Tres servicios}

Un geoportal, entendido como punto de entrada principal a la IDE de un país o región, debe dar acceso a los tres servicios web (Sánchez et al., 2012) que se consideran básicos en una IDE para que el usuario pueda satisfacer sus necesidades:

- Los servicios de visualización, por la importancia que tiene el análisis visual de los datos geográficos.

- El servicio de catálogo de metadatos, por ser el recurso clave para encontrar y organizar datos y recursos.

- Y el servicio de nomenclátor, como funcionalidad clave para encontrar un objeto geográfico y situarlo en un mapa. 
De 16 geoportales analizados, sólo cuatro cumplen este criterio, siete ofrecen acceso a dos servicios de los tres mencionados y cinco dan acceso a un solo tipo de servicio, el de visualización (Figura 6).

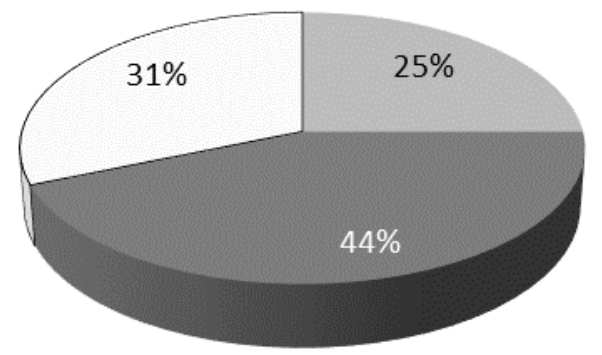

Geoportales con los tres
servicios básicos
Geoportales con dos
servicios básicos
Geoportales con un
servicio básico

Figura 6. Servicios básicos en los geoportales del continente americano.

\title{
Estandarización
}

Un geoportal de referencia debe acceder a los servicios web a través de interfaces estándar OGC (López-Pellicer et al., 2012), especialmente en los servicios más usados y extendidos, el Servicio Web de Mapas (OGC WMS, 2006), que es además norma ISO (ISO 19128), y su variante teselada WMTS (OGC WMTS, 2012).

Este punto se puede comprobar mediante cualquiera de las aplicaciones que captura y registra las peticiones que realiza el cliente en cada caso, como Httpwatch (Swenson, 2011), TamperData y otros sniffers.

Pues bien, de 16 geoportales analizados tan sólo ocho ofrecen acceso a servicios de visualización a través de las interfaces estándar OGC e ISO 19128, uno de ellos utiliza el estándar TMS de OSGeo, y los siete restantes (casi un 50\%) ofrecen servicios REST de ArcGIS (véase la Figura 7).

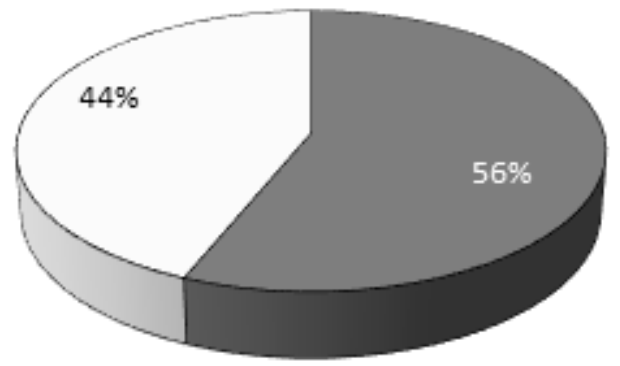

\author{
Geoportales con \\ Servicios WMS \\ $\square$ Geoportales con otros \\ servicios de \\ visualización
}

Figura 7. Acceso a los servicios de visualización a través de la interfaz estándar WMS en los geoportales del continente americano. 
Por otro lado, ocho de los 16 geoportales utilizan la cartografía de OpenStreetMap, servida con un servicio no OGC, como cartografía base.

\section{Aviso legal}

Cuando una organización publica en la web un recurso, ya sea un conjunto de datos, un servicio, una aplicación, una API o cualquier otro producto o servicio, debe adoptar una política de datos, mantenerla en el tiempo y darle la máxima difusión posible para establecer las reglas del juego de manera clara. Si es posible, se debe utilizar una de las licencias tipo (licencias estándar y públicamente disponibles en la red) Creative Commons, ${ }^{5}$ la denominada de Dominio Público ${ }^{6}$ (DP) y el Copyright (C), que se pueden combinar fácilmente entre sí, lo que facilita la llamada interoperabilidad de licencias.

En particular, en un geoportal deben aparecer en un lugar muy visible las condiciones de uso del geoportal, de los datos y de los servicios. Cuestión que no es habitual, ya que de los 16 geoportales considerados, tan sólo tres proporcionan alguna información relativa a las condiciones de uso de los recursos visibles. El 83\% restante no publica ninguna información referente a condiciones de uso y licencias.

\section{No logo}

Creemos que no se debe mencionar ningún nombre de marca, producto o empresa comercial ni en los nombres de los recursos, ni en sus URL, ni dentro del visualizador, ni en cualquier otro lugar que pueda suponer una promoción o publicidad indirecta, y por lo tanto un enrarecimiento del mercado. Ese tipo de información se puede dejar, por ejemplo en una pestaña o área de la página web del geoportal «Acerca de» por si hay alguien interesado en consultarla. Un ejemplo extremo sería llamar a una serie cartográfica oficial «Mapa de Hidrografía Microsoft 1: 400 000»; la misma idea puede aplicarse a los nombres, etiquetas y a la imagen en general de un geoportal y sus recursos. Hay que pensar que la URL (Uniform Resource Locator) de un recurso también es su URI (Uniform Resource Identifier) y quizás sea lo más natural utilizar cadenas de caracteres neutras.

En este criterio, de 16 geoportales, sólo seis mantienen una neutralidad razonable, el $62.5 \%$ restante (10 geoportales) muestran marcas comerciales, a veces de software propietario y a veces de software libre. Esas menciones se encuentran en las URL de los servicios, visualizadores o páginas web, o en el propio visualizador (véase la Figura 8).

$<$ http://es.creativecommons.org $>$.

$<$ https://es.wikipedia.org/wiki/Dominio_p\%C3\%BAblico>. 


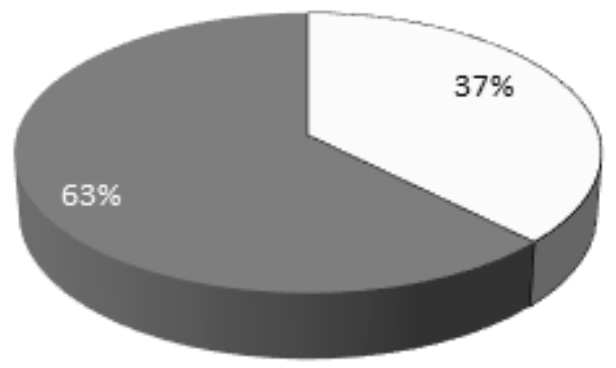

$\square$ Geoportales sin logos

- Geoportales con logos

Figura 8. Geoportales con logos, marcas o textos comerciales en las URL, el visualizador u otra ubicación fácilmente visible.

\section{Visibilidad de recursos}

Uno de los objetivos y responsabilidades fundamentales del geoportal de una IDE es dar visibilidad a los recursos interoperables de su ámbito. Para cumplir ese objetivo debe considerar como punto clave la publicación de las direcciones URL, no solo de los propios servicios web del nodo que alberga el geoportal, sino también las direcciones de los servicios disponibles en el ámbito de toda la IDE y las direcciones de los geoportales que componen la IDE en cuestión.

De 16 geoportales analizados, ocho publican las URL de los servicios y de los geoportales de la IDE correspondiente, tres sólo muestran los geoportales, dos muestran solo servicios y tres no muestran ningún recurso incluido en el ámbito de esa IDE (véase Figura 9.).

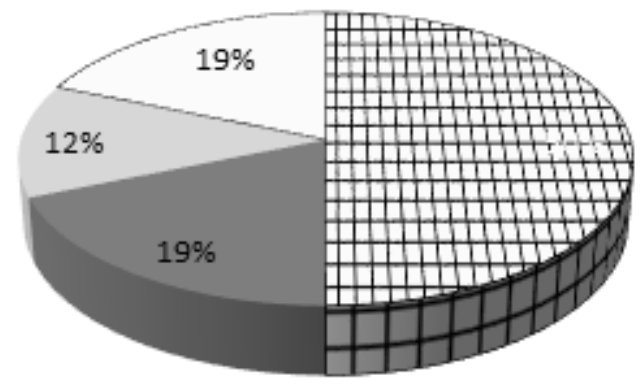

MURL de servicios y geoportales

URL de geoportales

URL de servicios

$\square \operatorname{Sin} U R L$

Figura 9. Visibilidad de los recursos disponibles en el geoportal principal de una IDE. 


\section{Difusión}

La difusión de un geoportal tiene una gran importancia para facilitar a los potenciales usuarios el que lo encuentren con facilidad (González, 2012). El administrador de un geoportal IDE debe asumir como una de sus responsabilidades, informar sobre su existencia y conseguir que los geoportales de referencia de ámbito mayor (como la web de GSDI, ${ }^{7}$ las páginas de UN-GGIM ${ }^{8}$ o el geoportal del Programa GeoSUR $)^{9}$ recojan al menos las direcciones URL de los servicios y un enlace para acceder al geoportal. Debido al amplio uso que se hace de portales de información generalista, nos parece aconsejable usarlos también para difundir el geoportal de una IDE. Por ejemplo, utilizar las versiones en inglés y en español de la Wikipedia u otros recursos similares.

Tomando como indicador de difusión el que las direcciones de los geoportales se encontraran recogidas en cuatro puntos de información:

- El geoportal del Programa GEOSUR

- En las entradas de IDE o Geoportal de la Wikipedia en español

- En las entradas de SDI o Geoportal de la Wikipedia en inglés

- En la lista de enlaces IDE de GSDI o en la lista de IDE recogidas en la web de UN-GGIM.

De los 16 geoportales analizados, cinco figuran sólo en una de esas listas, cinco aparecen en dos sitios y sólo seis están recogidos en tres o más de los lugares mencionados. El resto (más del 62\%) no ha hecho el esfuerzo suficiente para figurar en esos lugares que recopilan los geoportales e IDE del mundo (véase Figura 10).
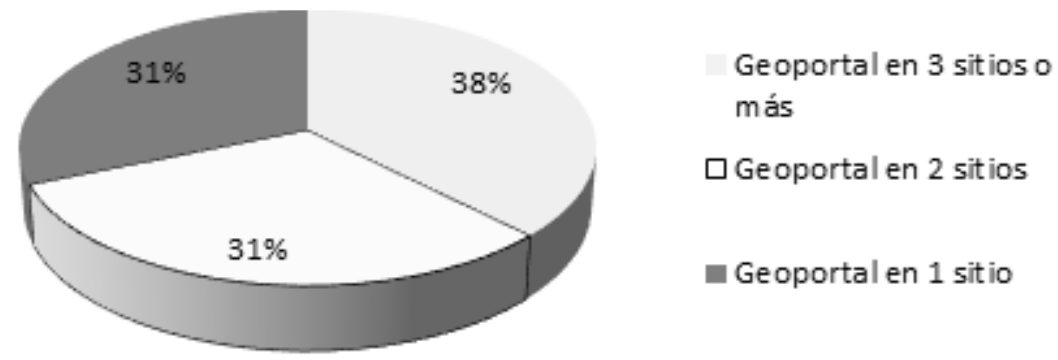

$\square$ Geoportal en 2 sitios

Geoportal en 1 sitio

Figura 10. Difusión basada en la publicación del enlace al geoportal en los sitios claves que recopilan esas URL.

$<$ http://gsdiassociation.org/index.php/publications/sdi-links.html >.

$<$ http://ggim.un.org/>.

$<$ https://www.geosur.info >. 


\section{Redes sociales}

Los geoportales adquieren mayor valor al incorporar a las redes sociales como otra herramienta de bajo coste para su promoción vía Internet (Alexopoulos et al., 2014; Maciel et al., 2016). En la actualidad, las redes sociales constituyen un mecanismo fundamental que contribuye decisivamente a la difusión de un proyecto y a obtener retroalimentación de los usuarios. Por esa razón parece importante que un proyecto IDE tenga presencia en las redes sociales más habituales, como Twitter, Facebook, Google+ e Instagram.

Una prospección de los 16 geoportales analizados arroja como resultado que siete de ellos sí tienen presencia en las redes sociales y los otros nueve, no la tienen.

\section{Disponibilidad}

Entre los requisitos necesarios para garantizar la calidad de un servicio web, la disponibilidad ocupa un lugar de importancia (Orantes y Botello, 2010). En el caso de un geoportal, entendido también de acuerdo a la norma ISO 19119 (2016) como un servicio que se ofrece en la red, es necesario considerar su disponibilidad, es decir, la probabilidad anual de que el geoportal no esté caído y responda, expresado en tanto por ciento.

Hay pocas referencias de valores mínimos o recomendados de este parámetro. Por ejemplo, los requisitos de disponibilidad que las Normas de Ejecución INSPIRE $^{10}$ establece para los servicios web son de al menos un $99 \%$, si se toma esta referencia para geoportales, supondría menos de 88 horas, es decir 36.5 días, de inactividad cada año.

No se ha tenido ocasión ni tiempo para realizar un estudio riguroso y completo, que pasaría por lanzar peticiones regulares (en las Normas de Ejecución INSPIRE se establece que cada 6 minutos) durante largos periodos de tiempo (un año como mínimo). Sin embargo, durante las dos semanas que hemos estado realizando pruebas, los 16 geoportales han respondido, por lo que suponemos que no hay grandes problemas de disponibilidad.

\section{Rendimiento}

Para completar una primera descripción de la calidad del servicio que consiste en ofrecer un geoportal al usuario, habría que considerar el rendimiento, entendido como el tiempo de respuesta o tiempo transcurrido en segundos desde que el ordenador del usuario lanza una petición hasta que se completa la respuesta correspondiente (Quiroz, 2015). 
El internauta se comporta a menudo de manera impaciente, por lo que interesa mantener tiempos de respuesta inferiores a 10 segundos para peticiones pesadas, como abrir un visualizador, y de 3 segundos para peticiones sencillas, como abrir o refrescar una página web, teniendo en cuenta que para el $10 \%$ de los usuarios es recomendable que sea menor de 5 segundos (Córdoba, 2017).

De nuevo, este punto se puede comprobar mediante cualquiera de las aplicaciones que captura y registra las peticiones que realiza el cliente en cada caso, como Httpwatch, TamperData y otros sniffers, que registran los tiempos de respuesta. Hay que tener en cuenta que dichos tiempos de respuesta dependen de múltiples factores, como la potencia del ordenador del usuario, su ancho de banda y el tráfico en la red en cada momento. Tales circunstancias no son siempre achacables al geoportal en cuestión, por lo que para determinar este aspecto habría que definir unas condiciones estándar.

Este parámetro se ha determinado al mismo tiempo que se evaluaba el correcto funcionamiento en los cuatro navegadores más conocidos, abriendo a la vez la página principal y el visualizador de cada geoportal en los cuatro navegadores, lo que genera una pequeña concurrencia, de seis a siete de la mañana, hora de Madrid, es decir de una a dos de la mañana (hora del huso - 4), cuando hay poco tráfico. Se han tomado como tiempos de respuesta admisibles, $3 \mathrm{~s}$ para abrir o refrescar una página web y $10 \mathrm{~s}$ para arrancar el visualizador.

De los 16 geoportales analizados, la mayoría (14), han mostrado un buen comportamiento y sólo dos, han resultado demasiado lentos.

\section{Retroalimentación}

Un geoportal de una IDE debe ofrecer mecanismos y vías de comunicación para recibir quejas, sugerencias y opiniones de su comunidad de usuarios (Calderón et al., 2014). El mantener canales de retroalimentación es especialmente importante porque dado cómo funciona y el diseño de las interfaces estándar, el usuario IDE es esencialmente anónimo y resulta muy interesante conocer su opinión para satisfacer mejor sus necesidades.

En este punto, de 16 geoportales analizados, 12 ofrecen canales de retroalimentación notables, y sólo cuatro (un 25\%) carecen de ellos (véase la Figura 10). 


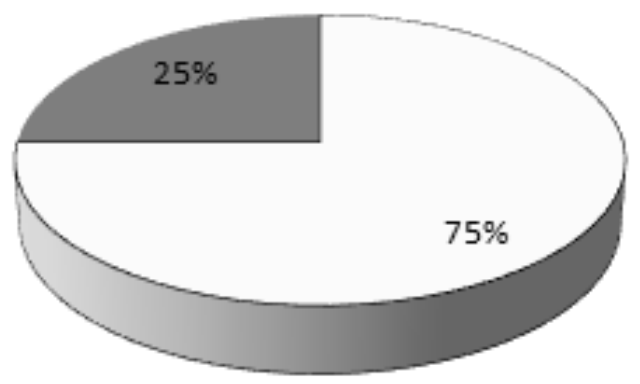

$\square$ Geoportales con canales de retroalimentación

Geoportal sin canales de retroalimentación

Figura 10. Geoportales que ofrecen a los usuarios canales para que expresen sus peticiones, dudas y quejas.

\section{Resumen de resultados}

La situación general que hemos detectado puede sintetizarse en una tabla, que muestra los 19 indicadores derivados de los aspectos considerados y un valor que refleja el número de geoportales que se estima satisfacen cada uno de los criterios, aunque sea de forma parcial (véase la Tabla 2 y la Figura 11).

Por último hay que decir que hay un aspecto problemático que no se puede evaluar ahora y es la persistencia a largo plazo de los geoportales. En el blog de la IDEE se han recopilado las direcciones de los geoportales del continente americano en tres ocasiones, en los años 2008, 2012 y 2016, en sendas entradas. De los ocho geoportales recogidos en el 2008, sólo cinco, el 62.5\%, responden hoy en día, y de los 14 reflejados en el 2012, actualmente siguen respondiendo tan solo ocho, el $57.1 \%$.

Se trata de un aspecto más político y organizativo que técnico, pero que puede ser un inconveniente a largo plazo. Una posible solución es establecer un sistema de identificadores permanentes de recursos que se mantenga en el tiempo.

Se puede definir un indicador global para cada geoportal asignando un peso a cada uno de los 19 criterios normalizados. Si se considera que los más relevantes (Apertura, Multilingüismo, Responsividad, Identidad, Accesibilidad, Usabilidad, Tres Servicios, Estandarización, Aviso legal, Visibilidad de recursos, Disponibilidad y Rendimiento) deben tener un peso 10, que sea el doble del peso asignado a los menos relevantes (Navegadores, Autoidentificación, Dominio, Accesibilidad, No logo, Difusión y Redes sociales) que tendrán un peso de 5, se asignan puntuaciones de 0 a 10 a cada geoportal en cada criterio, se hallan las sumas ponderadas para todos ellos y se normaliza el resultado a 100, para que resulte más intuitivo, se obtiene la distribución de valores que puede verse en la Figura 12. 
Tabla 2

Criterios contemplados y número de geoportales que los satisfacen

\begin{tabular}{lcclcc}
\hline \multicolumn{1}{c}{ Criterio } & $\begin{array}{c}\text { Valor } \\
\text { (máx. 16) }\end{array}$ & $\begin{array}{c}\text { Tanto por } \\
\text { ciento }\end{array}$ & Criterio & $\begin{array}{c}\text { Valor } \\
\text { (máx. 16) }\end{array}$ & $\begin{array}{c}\text { Tanto por } \\
\text { ciento }\end{array}$ \\
\hline Apertura & 16 & $100 \%$ & Estandarización & 9 & $56 \%$ \\
Multilingüismo & 3 & $19 \%$ & Aviso legal & 3 & $19 \%$ \\
Responsividad & 14 & $88 \%$ & No logo & 6 & $38 \%$ \\
Navegadores & 16 & $100 \%$ & Visibilidad & 8 & $50 \%$ \\
Autoidentificación & 13 & $81 \%$ & Difusión & 6 & $38 \%$ \\
Identidad & 15 & $94 \%$ & Redes sociales & 7 & $44 \%$ \\
Dominio & 16 & $100 \%$ & Disponibilidad & 16 & $100 \%$ \\
Accesibilidad & 1 & $6 \%$ & Rendimiento & 14 & $88 \%$ \\
Usabilidad & 6 & $38 \%$ & Retroalimentación & 12 & $75 \%$ \\
Tres servicios & 4 & $25 \%$ & & & \\
\hline
\end{tabular}

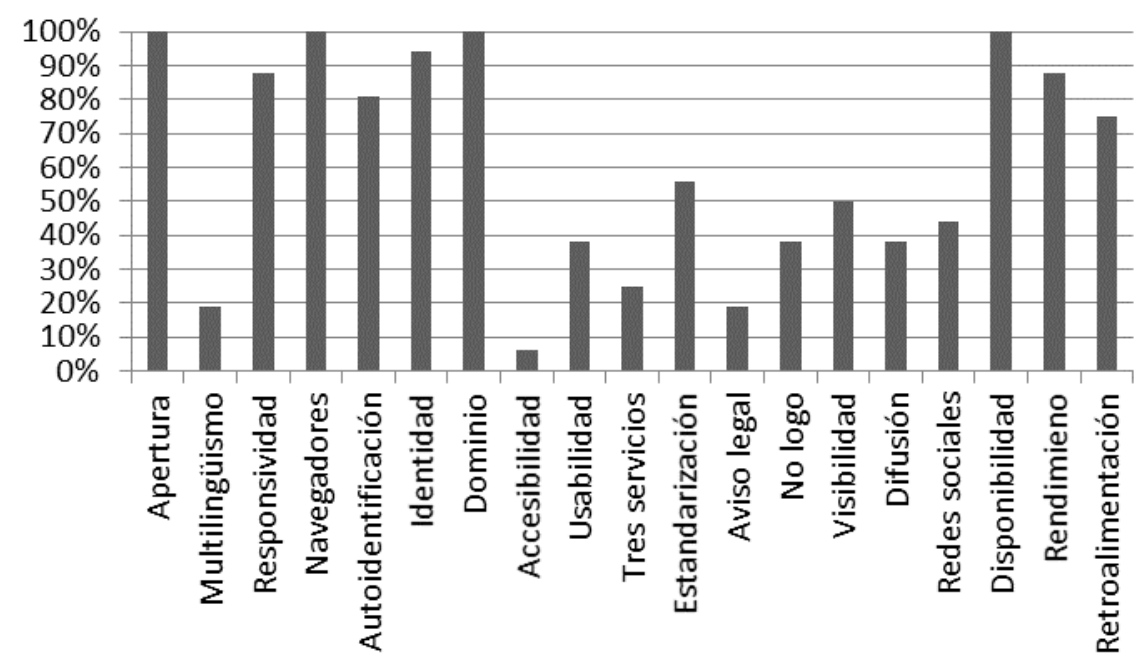

Figura 11. Tanto por ciento de geoportales que satisfacen cada uno de los criterios elegidos de interoperabilidad práctica. 


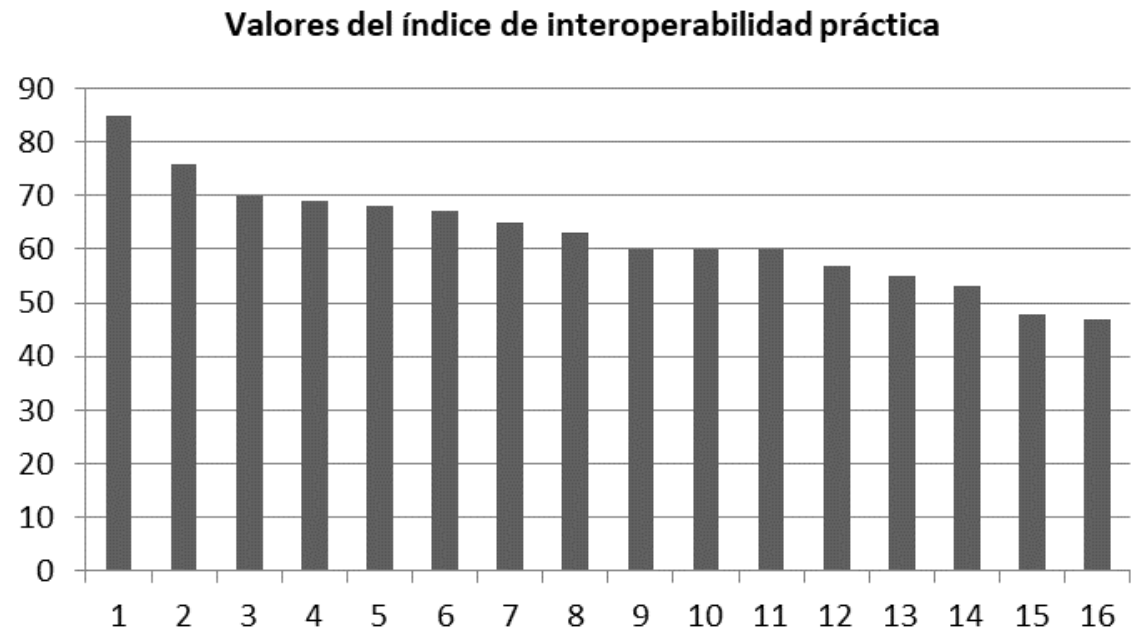

Figura 12. Distribución de valores del índice de interoperabilidad práctica para cada geoportal, de 0 a 100 y ordenados de mayor a menor.

Se puede ver que hay tres geoportales por encima de 70, un grupo de ocho entre 60 y 70, a continuación otros tres que están entre 50 y 60 y solo dos ligeramente por debajo de 50. El valor medio es de 63.

\section{Conclusiones}

Según el grupo de expertos consultados, los resultados se adecúan a lo esperado en los criterios de Apertura, Navegadores, Dominio y Disponibilidad. Se resalta que la calidad del servicio que supone ofrecer un geoportal en la web, descrita mediante la Disponibilidad y el Rendimiento, es también conforme a lo deseado.

Aspectos como la Responsividad, Identidad y Retroalimentación están en general satisfactoriamente resueltos. Sin embargo, hay otros muy esenciales que en muchos geoportales necesitan mejorarse, independientemente del coste implicado, como la Estandarización, No logo, la Usabilidad o los Tres servicios.

En cuanto al uso de OpenStreetMap como mapa base en siete geoportales, probablemente se deba a que ofrece cobertura universal y alta calidad de servicio, en cuanto a rendimiento y disponibilidad.

Los aspectos cuya mejora supondría una mayor inversión de recursos (como la Disponibilidad, el Rendimiento y la Responsividad) están en general resueltos, mientras que otros bastante sencillos de mejorar con una inversión muy moderada, no lo están, especialmente el Multilingüismo, la Accesibilidad, el Aviso legal, la Visibilidad de recursos, la Difusión y las Redes sociales. 
Si se comparan estos resultados con los de estudios anteriores acerca de los nodos IDE de España (Rodríguez et al., 2015), el panorama de desarrollo e implementación de IDE en la región es comparable al estado de desarrollo de prácticamente cualquier otro continente o región, siendo los problemas y dificultades a ambos lados del Atlántico, muy similares.

Debido a que las IDE constituyen una tecnología, novedosa, en cuya implementación confluyen muchos factores, algunos organizativos que son los más difíciles de solucionar y coordinar, se considera que la situación general ha evolucionado positivamente. Hay cuestiones interesantes en diferentes nodos IDE, lo que debe servir para generar la motivación necesaria para mejorar y llevar a la práctica la filosofía de la mejora continuada.

Posibles líneas de trabajo futuro podrían ser:

- Contrastar la pertinencia y completitud de los criterios mencionados con una población de expertos en la materia y con los usuarios.

- Establecer un observatorio IDE que evaluase los criterios propuestos de manera periódica y publicase los resultados.

\section{Bibliografía}

Alexopoulos, C.; Zuiderwijk, A.; Charapabidis, Y.; Loukis, E. and Janssen, M. (2014, September). "Designing a second generation of open data platforms: Integrating open data and social media”, International Conference on Electronic Government, Springer Berlin Heidelberg, pp. 230-241.

Béjar, R.; Latre, M.A.; Nogueras, J.; Muro, P.M.; Zarazaga, F.J. (2009). "Systems of Systems as a Conceptual Framework for Spatial Data Infrastructures”, International Journal for Spatial Data Infrastructures Research, vol. 4, pp. 201-207.

Bernabé-Poveda, M.A. and González, M.E. (2014). "Sobre la necesaria usabilidad de los geoportales como puertas de entrada a las IDE”, Geofocus. Revista Internacional de Ciencia y Tecnología de la Informacion Geográfica, vol. 1, núm. 14, pp. 1-5.

Calderón, L.J.; Campoverde, J.Y. y Hoehne, A.V. (2014). "El usuario como factor de éxito en el diseño de un geoportal”, Geofocus. Revista internacional de Ciencia y Tecnología de la Información Geográfica, núm. 14, pp. 181-210.

Blog-IDEE, “Las IDE de América”, 2016. Disponible en $<$ http://blog-idee.blogspot.com.es/2016/12/las-ide-de-america.html>, consultado el 13 de marzo de 2017.

Blog-IDEE, "Las IDE de América Latina y Caribe", 2012. Disponible en $<$ http://blog-idee.blogspot.com.es/2012/01/las-ide-de-america-latina-y-el-caribe. html>, consultado el 13 de marzo de 2017. 
Blog-IDEE, "Las IDE en Latinoamérica” (2008). Disponible en <http://blogidee.blogspot.com.es/2008/07/las-ide-en-latinoamrica.html>, consultado el 13 de marzo de 2017.

BOE (2010). "Real Decreto 4/2010 por el que se establece el Esquema Nacional de Interoperabilidad en el Ámbito de la Administración Electrónica”, disponible en $<$ http://www.boe.es/buscar/doc.php?id=BOE-A-2010-1331>, consultado el 12 de marzo de 2017.

Comisión Europea (2007). "Directiva 2007/2/CE INSPIRE". Disponible en $<$ http://eur-lex.europa.eu/LexUriServ/LexUriServ.do?uri=OJ:L:2007:108:0001: 0014:es:PDF>, consultado el 13 de marzo de 2017.

(2004). "European Interoperability Framework" v1.0, disponible en <http://ec.europa.eu/idabc/servlets/Docd552.pdf?id=19529>, consultado el 13 de marzo de 2017.

(2017). "European Interoperability Framework" v2.0, disponible en $<$ http://eur-lex.europa.eu/resource.html?uri=cellar:2c2f2554-0faf-11e7-8a3501aa75ed71a1.0017.02/DOC_3\&format=PDF $>$, consultado el 29 de marzo de 2017.

Córdoba, J.; Cachero, C.; Calero, C.; Genero, M. y Marhuenda, Y. (2007). "Modelo de Calidad para Portales Bancarios", XXXIII Conferencia Latinoamericana de Informática (CLEI’07), disponible en <http://www.dlsi.ua.es/ ccachero/papers/ clei07.pdf $>$.

Freire, F.C. (2010). "Los nuevos modelos de gestión de las empresas mediáticas/New business models of Media management”, Estudios sobre el mensaje periodístico, núm. 16, pp. 13-31.

González, M.E. (2012). "Las Infraestructuras de Datos Espaciales (IDE) como un recurso educativo TIC. Estrategias de formación y difusión para el profesorado de la Educación Secundaria Obligatoria”, Doctoral dissertation, Topografía, disponible en $<$ http://oa.upm.es/11015/>.

GSDI (2012). SDI Cookbook, disponible en http://gsdiassociation.org/images/ publications/cookbooks/SDI_Cookbook_fromWiki_2012_update.pdf, consultado el 13 de marzo de 2017.

ISO 19119 (2016). "ISO 19128:2016 Geographic Information - Services”.

ISO 19128 (2005). "ISO 19128:2005 Geographic Information - Web Map Server Interface".

ISO 19133 (2005). "ISO 19133:2005 Geographic Information - Location- based service - Tracking and navigation".

ISO 19134 (2007). "ISO 19134:2007 Geographic Information - Location- based service - Multimodal routing and navigation”.

ISO 19142 (2010). "ISO 19142:2010 Geographic Information - Web Feature Service". 
ISO/IEC (2001). "ISO 9126-1: 2001 Software engineering - Product quality - Part 1: Quality model”.

ISO/IEC (1998). "ISO/IEC 9241-11:1998 Ergonomic requirements for office work with visual display terminals - Part 11: Guidance on usability".

ISO/IEC (1993). "ISO/IEC 2382-1:1993 Information Technology - VocabularyPart 1: Fundamental Terms".

LISIGE. (2010). "Ley 14/2010 de las Infraestructuras y Servicios de Información Geográfica en España” (LISIGE), disponible en $<$ https://www.boe.es/buscar/act.php?id=BOE-A-2010-10707>, consultado el 13 de marzo de 2017.

Lopez-Pellicer, F.J.; Barrera, J.; Abad, P.; Sánchez, A.; López, E. y Muro-Medrano, P.R. (2012). "Una aproximación ágil al problema de la conformidad de servicios con INSPIRE”, presentado en Actas de las III Jornadas Ibéricas de Infraestructuras de Datos Espaciales JIIDE, Madrid.

Maciel, A.M.; Valenzuela, K.V.R. y Flores, J.M.V. (2016). "Los geoportales, una herramienta alternativa para el desarrollo económico local. El caso del SIGUE Vallarta”, Paakat. Revista de Tecnología y Sociedad, vol. 6, núm. 11.

Manrique Sancho, M.T. y Manso Calleja, M.A. (2012). "Los Geoportales. Perspectivas desde la usabilidad”, en Bernabé Poveda, M.A, y López Vázquez, C.M. (eds.), Fundamentos de las Infraestructuras de Datos Espaciales, Madrid, UPM Press, pp. 393-402.

Open Geospatial Consortium (2012). "Sensor Observation Service Interface Standard" 2.0, disponible en <http://www.opengeospatial.org/standards/sos>, consultado el 3 de marzo de 2017.

(2006). “Web Map Service Implementation Specification” 1.3.0, disponible en <http://www.opengeospatial.org/standards/wms $>$, consultado el 3 de marzo de 2017.

_ (2010) "Web Map Tile Service Implementation Standard” 1.1.0, disponible en <http://www.opengeospatial.org/standards/wmts>, consultado el 3 de marzo de 2017.

— Open Geospatial Consortium (2010). "Web Service Common Implementation Specification", 2.0.0, disponible en <http://www.opengeospatial.org/ standards/common>, consultado el 13 de marzo de 2017.

Orantes Jiménez, S.D. y Botello Castillo, A. (2010). "Calidad y disponibilidad en los servicios Web”, Revista Digital Universitaria, núm. 3, Universidad Nacional Autónoma de México (UNAM), México, pp. 1-9.

Quiroz Sani, L.Á. (2015). Análisis comparativo de servidores web de mapas Georeferenciados Open source para la implementación de los servidores WMS y EFS del Geoportal del CONAGOPARE, Universidad Nacional de Chimborazo. 
Rodríguez, A.F.; Cevidanes, A.; Sánchez, A.; Abad, P. y Juanatey, M. (2015). “Interoperabilidad práctica de los geoportales de la IDEE”, en Mapping Interactivo, vol. 175, Madrid, España, pp. 8-16.

Rodríguez, A.F., Abad, P. et al. (2009). "Towards a Set of Recommendations about How to Implement Geoportals in SDI Nodes”, comunicación en la 24 Conferencia Cartográfica Internacional, Santiago de Chile, noviembre 15-21.

Rodríguez, A.F. (2008) "Conclusiones del Curso IDE de AECI, UPM e IGN sobre implementación de Geoportales IDE”, Newsletter IDE de Iberoamérica, vol. 4, núm. 8, <http://redgeomatica.rediris.es/newsletter/Newsletter_v4_08.pdf>, consultado el 13 de marzo de 2017.

Sánchez Maganto, A.; Rodríguez Pascual, A.F. y Bernabé Poveda, M.A. (2012). “Componentes de una IDE”, en Bernabé Poveda, M.A. y López Vázquez, C.M. (eds.), "Fundamentos de las Infraestructuras de Datos Espaciales”, Madrid, UPM Press, pp. 55-66.

Swenson, E. (2011). Tools and Resources. Practical SharePoint 2010 Branding and Customization, pp. 205-218. 